\title{
Guideline-recommended Medications and Physical Function in Older Adults with Multiple Chronic Conditions
}

\author{
Gail McAvay, PhD ${ }^{1}$, Heather G. Allore, PhD. ${ }^{1,2}$, Andrew B. Cohen, MD, PhD ${ }^{1}$, Danijela \\ Gnjidic, $\mathrm{PhD}^{3}$, Terrence E. Murphy, $\mathrm{PhD}^{1,2}$, and Mary E. Tinetti, $\mathbf{M D}^{1}$ \\ ${ }^{1}$ Department of Internal Medicine, Yale School of Medicine, New Haven, Connecticut \\ ${ }^{2}$ Department of Biostatistics, Yale School of Public Health, New Haven, Connecticut \\ ${ }^{3}$ Faculty of Pharmacy and Charles Perkins Centre, University of Sydney, Sydney, Australia
}

\begin{abstract}
Background-The benefit or harm of a single medication recommended for one specific condition can be difficult to determine in patients with multiple chronic conditions and polypharmacy. There is limited information on the associations between guideline-recommended medications and physical function in older adults with multiple chronic conditions.
\end{abstract}

Objectives-To estimate the beneficial or harmful associations between guideline-recommended medications and decline in physical function in older adults with multiple chronic conditions.

Design-Prospective observational cohort.

Setting一National

Participants-Community dwelling adults aged 65 and older from the Medicare Current Beneficiary Survey study ( $\mathrm{N}=3273)$. Participants with atrial fibrillation, coronary artery disease, depression, diabetes or heart failure were included.

Measurements-Self-reported decline in physical function, guideline-recommended medications, polypharmacy (taking fewer than 7 versus 7 or more concomitant medications), chronic conditions, socio-demographic, behavioral, and health risk factors.

Results-The risk of decline in function in the overall sample was highest in participants with heart failure $(35.4 \%, 95 \%$ confidence interval $(\mathrm{CI})=26.3-44.5)$ and lowest for those with atrial fibrillation $(20.6 \%, 95 \% \mathrm{CI}=14.9-26.2)$. In the overall sample, none of the six guidelinerecommended medications was associated with decline un physical function across the five study conditions, although in the group with low polypharmacy exposure, there was lower risk of decline in those with heart failure taking renin angiotensin system blockers (hazard ratio $(\mathrm{HR})=0.40$,

Correspondence to: Gail McAvay, Ph.D., Department of Internal Medicine, Yale School of Medicine, 300 George St, Suite 775, New Haven, CT 06511-6624, Tel: 203-737-1964, Fax: 203-785-4823, Gail.McAvay@yale.edu.

Conflict of Interest: None of the authors has any relationships with industry or any actual or potential conflicts of interest related to this project.

Author Contributions: Study concept and design: MT, HA. Acquisition of data: GM. Analysis and interpretation of data: GM, HA, MT, AC, DG, TM. Preparation of manuscript: GM, HA, MT, AC, DJ, TM.

Sponsor's Role: The funding organization had no role in the design or conduct of the study; acquisition, management, analysis or interpretation of the data; or preparation, review, or approval of the manuscript. 
95\% $\mathrm{CI}=0.16-0.99)$ and greater risk of decline in physical function for participants with diabetes taking statins $(\mathrm{HR}=2.27,95 \% \mathrm{CI}=1.39-3.69)$.

Conclusions-In older adults with multiple chronic conditions, guideline-recommended medications for atrial fibrillation, coronary artery disease, depression, diabetes and heart failure were largely not associated with self-reported decline in physical function, although associations for some medications were present in those with a lower polypharmacy exposure.

\section{Keywords}

Guideline-recommended medications; physical function; multiple chronic conditions; polypharmacy

\section{INTRODUCTION}

Nearly three quarters of adults aged 65 and over have multiple chronic conditions (1). Prescribing decisions for these persons tend to be made using clinical practice guidelines for individual conditions (2). Consequently, persons with multiple conditions take large numbers of medications (3). It is well established that taking many medications is burdensome to patients increasing the likelihood of adverse drug effects $(4,5)$. Furthermore, the benefit or harm of a medication prescribed for a single condition can be difficult to determine for a patient with multiple conditions (6).

A majority of older adults identify physical function as their most important health outcome when faced with trade-offs regarding the benefits and harms of medications (7). Understanding the effects of medications on multidimensional measures of function is therefore necessary in improving medication decision-making.

Given the limited data on the benefits or harms of commonly prescribed guideline medications used among older adults with multimorbidity and polypharmacy, we examined the association between beta-blockers, non-dihydropyridine calcium channel blockers (CCB), renin angiotensin system blockers (RAS blockers), statins, metformin and selective serotonin reuptake inhibitors (SSRI) and physical function among a national sample of older adults with multiple chronic conditions. Potential benefits of these medications on physical function could occur via improvements in muscle strength $(8,9)$, reductions in inflammation $(10,11)$ or improved glycemic control $(12)$. Conversely, potentially harmful effects on physical function could occur due to muscle weakness, $(13,14)$ and orthostatic hypotension $(15,16)$.

We studied participants with at least one of five symptomatic conditions for which these medications are indicated, specifically atrial fibrillation, coronary artery disease (CAD), depression, Type 2 diabetes and heart failure. As polypharmacy has been shown to increase the likelihood of adverse drug events, hospitalization, falls, functional decline and mortality, we estimated the association between each guideline medication and physical function stratified by polypharmacy. $(5,17)$. 


\section{METHODS}

\section{Study Population}

The combined sample included participants in the Medicare Current Beneficiary Survey (MCBS) study from five yearly panels drawn in 2005-2009. Each panel is followed for three years. The current study had follow-up data available from 2006 to 2011. MCBS is a nationally representative sample of Medicare beneficiaries obtained from the Centers for Medicare and Medicaid Services enrollment file (18).

Details on the study population are described elsewhere (19-21); briefly, we identified common chronic conditions ( $>=10 \%$ prevalence) associated with mortality and for which there is at least one oral prescription drug recommended by disease guidelines and used by at least $10 \%$ of the final study cohort. The final conditions included atrial fibrillation, coronary artery disease, depression, Type 2 diabetes mellitus, heart failure, hyperlipidemia, and hypertension. Conditions were identified by at least one inpatient or two other types of claims (outpatient, physician, skilled nursing, home health).

Participants for this study included those age 65 and over who had one or more of five common symptomatic chronic conditions including atrial fibrillation, CAD, depression, Type 2 diabetes, heart failure (referred to hereafter as study conditions), and at least one other of the following chronic conditions: hyperlipidemia, hypertension, thromboembolic disease or chronic kidney disease (referred to hereafter as coexisting conditions). We consider these five conditions as symptomatic because treatment is focused on affecting individuals' current functioning, whereas with asymptomatic conditions the focus is on prevention of future outcomes. (22).

Of 19,043 MCBS community-dwelling participants, 2682 were Medicare Advantage participants lacking claims data, 9141 did not meet the condition criteria, 1505 were nonrespondents, 277 lacked medication data at study entry leaving 5438 participants. We also excluded 2165 participants who used equipment to walk or transfer from a bed or chair for a total sample size of 3273 . The study was deemed exempt by the Yale University Human Investigation Committee because it involved existing, publicly-available, de-identified data.

\section{Outcome}

At baseline and each annual follow-up, self-reported physical function was assessed by asking participants to rate their current level of difficulty in performing five activities (writing/handling objects, extending arms above shoulder, stooping/kneeling/crouching, lifting/carrying 10 pounds, walking $1 / 4$ mile or $2-3$ blocks) (23). We created a count variable $(0-5)$ reflecting the number of activities the participant was able to perform (at any level of difficulty) versus being completely unable to perform the activity. We defined the outcome "decline in function" as a decrease in the number of activities the participant was able to perform at follow-up. Participants who were unable to perform all 5 activities at baseline were excluded from the analysis $(n=8)$. Many studies have reported outcomes of acquiring an additional disability (change of 1 point) as a clinically meaningful change (24). 


\section{Exposures}

Medications-Commonly used medications listed in recent national disease guidelines for the five study conditions were examined (25-29). These medications have biological mechanisms for an adverse or beneficial effect on current physical function via muscle strength, (30, 31), inflammation $(32,33)$, insulin resistance $(34,35)$, arrhythmias $(36,37)$ and orthostatic hypotension (38). They include beta-blockers (cardio selective or alpha/betablockers) (39) for atrial fibrillation, CAD, and heart failure; non-dihydropyridine CCB for atrial fibrillation (14); RAS blockers for CAD, diabetes, and heart failure $(8,9)$; statins for CAD and diabetes $(13,14)$; metformin for diabetes (40); and selective serotonin reuptake inhibitors (SSRI) for depression (16). Prescription medications were ascertained by direct observation of medication containers at the baseline and annual follow-up face-to-face interviews. Changes in medications were accounted for by updating the measures of prescription drug use annually. A polypharmacy measure was created based on the number of prescription concomitant medications (excluding the guideline-recommended medications under study) the participant was taking at baseline. A dichotomous measure of polypharmacy was defined as low polypharmacy, for those taking fewer than 7 prescription concomitant medications (the mean) versus those taking 7 or more prescription concomitant medications, the high polypharmacy group.

Covariates-Socio-demographic, behavioral, and health covariates were selected based on previously identified risk factors (41) and characteristics associated with medication exposure. They included age, gender; race (non-white vs. white); Hispanic ethnicity; income $<\$ 25,000$ a year; number of concomitant prescription medications; prescription drug insurance coverage; days spent in the hospital in the year preceding follow-up (categories: 0, 1-6, >=7); smoking; obesity (body mass index > 30); hearing; vision and cognitive impairments. Hearing impairment was based on "a lot of trouble hearing" or deaf and vision impairment was based on "a lot of trouble seeing" or blindness. Cognitive impairment was defined as a dementia or cognitive disorder claim, or self-reported memory loss plus either trouble concentrating or difficulty making decisions interfering with activities of daily living. Co-existing study conditions hyperlipidemia, hypertension, thromboembolic disease, chronic kidney disease, in addition to osteoarthritis, and Elixhauser comorbidity scale (42) were included in the analyses. The guideline recommendation for atrial fibrillation is limited to non-dihydropyridine CCB. We also included dihydropyridine CCB as a covariate rather than reduce our sample by excluding participants taking dihydropyridine CCB. Finally, as use of insulin may reflect more severe cases of diabetes, we included this as a covariate in the analyses of participants with diabetes.

\section{Statistical Analysis}

To limit the estimation of associations between each guideline-recommended medication and decline in physical function to those with the condition for which the medication is indicated, five separate analytic samples were created for participants with atrial fibrillation, $\mathrm{CAD}$, depression, diabetes and heart failure. Baseline characteristics were summarized as frequencies and percentages, or means and standard errors. The product-limit method was used to estimate the unadjusted proportion of participants who declined over follow-up. As MCBS had annual interviews, an interval-censored time-to-event analysis using a 
complementary log-log link, was estimated, with repeated observations for participants until the time of decline, drop-out or end of follow-up whichever occurred first. For each study condition model, time-varying main effects for the medications indicated by guidelines for the condition were updated along with adjustment for the other co-existing conditions, medications and covariates. These models yield hazard ratios (HR) of the association of each guideline-recommended medication and decline in physical function. Analyses for the overall sample and with stratification by polypharmacy were conducted. Interaction terms for polypharmacy group by guideline medication were included in the model to test for differences by polypharmacy in the association between each study medication and decline in function.

Given the discrete time intervals, the competing risk of death could not be modeled using methods for continuous time. Instead, we performed sensitivity analyses by imputing the five missing function items for the deceased under assumptions of both missing at random (MAR) and missing not at random (MNAR). For MNAR, we examined the effect of adding bias by increasing the proportion who were unable to perform each item among the values imputed for decedents, using the SAS PROC MI procedure (43).

Multiple imputation with 10 replicates was used for missing medication (at follow-up) which was $<1 \%$, and for covariate data, which was $<1.9 \%$, using SAS/STAT ${ }^{\circledR}$ PROC MI and MIANALYZE. We used SAS Version 9.4 (SAS Institute Inc., Cary, NC) with p-values of 0.05 (two-tailed) used to denote statistical significance.

\section{RESULTS}

Characteristics of the participants in the five study condition groups are shown in Table 1. As participants could have multiple study conditions, we do not statistically compare groups. The most prevalent chronic conditions were hypertension (90.0-95.9\%), hyperlipidemia (77.3-91.2\%) and osteoarthritis (51.5-59.7\%), and the average number of Elixhauser comorbidities was highest in those with heart failure (Mean=2.6, SE=.08), and lowest in those with diabetes (Mean=1.3, SE=.04). The mean number of concomitant medications ranged from $6.6(\mathrm{SE}=.17)$ in those with atrial fibrillation to an average of $8.2(\mathrm{SE}=.21)$ in those with heart failure.

Multimorbidity was common. The majority of participants with heart failure and atrial fibrillation had CAD, $68.6 \%$ and $54.2 \%$ respectively, whereas diabetes co-occurred with the other conditions in approximately one-third of participants (32.1-45.3\%).

More than $78 \%$ of the participants received at least one guideline-recommended medication, with the exception of SSRIs (40.8\%) (Table 2). Percentages receiving the individual medications recommended for each condition, ranged from $15.2 \%$ for non-dihydropyridine CCB to $71.8 \%$ for beta-blockers.

The majority of participants were able to perform all five activities at baseline, ranging from $77.9 \%$ to $85.0 \%$ across study condition (Table 2 ). When stratified by polypharmacy, participants in the high polypharmacy group were less likely to be able to perform all five activities at baseline. 
The percentage of those who declined in the overall sample, and stratified by polypharmacy, is shown at the bottom of Table 2. In the overall sample, decline was highest in those with heart failure (35.4\%; $\mathrm{CI}=26.3-44.5)$ and lowest for participants with atrial fibrillation (20.6\%; $\mathrm{CI}=14.9-26.2)$. When the sample was stratified by polypharmacy, decline was substantially greater for those in the high polypharmacy group among participants with $\mathrm{CAD}$, diabetes and heart failure. Importantly, although participants in the high polypharmacy group were more disabled at baseline and subsequently had less chance of experiencing a decline, decline was greater for those taking more medications with the exception of depressed participants.

Figure 1 displays the adjusted hazard ratios (aHR) for each medication by study condition, reflecting the risk of decline in physical function over follow-up for those taking the medication relative to those not taking the medication. There were no statistically significant associations between any of the guideline-recommended medications and risk of decline in function.

The results stratified by low and high polypharmacy are displayed in Figure 2. There were two significant interactions reflecting differences in the association between medication and function by polypharmacy. Among participants with heart failure in the low polypharmacy group, RAS blockers were associated with reduced risk of decline in function (aHR=.40, $\mathrm{CI}=0.16-0.99)$ versus no association in the high polypharmacy group $(\mathrm{aHR}=1.14, \mathrm{CI}=.67-$ 1.95). The interaction of polypharmacy and statins was also significant, with an increased risk of decline among diabetics in the low polypharmacy group ( $\mathrm{aHR}=2.27, \mathrm{CI}=1.39-3.69)$ versus no association in the high polypharmacy group ( $\mathrm{aHR}=.77, \mathrm{CI}=.54-1.10)$. The hazard ratio for statins remained significant ( $\mathrm{aHR}=2.09, \mathrm{CI}=1.24-3.52$ ), when excluding insulin users. Finally, to check the sensitivity of the results to the cut-point used for polypharmacy, the same interactions were significant using the continuous number of concomitant medications.

We considered the possibility that differences in baseline function status might influence these findings. When we limited the analysis to those who could perform all five tasks at baseline the findings did not meaningfully differ. For example, the interactions between polypharmacy and RAS blockers in heart failure participants (interaction p-value $=.02$ ) and statins in diabetic patients (interaction $\mathrm{p}$-value $=.01$ ) remained significant. The adjusted hazard ratio for RAS blockers was $0.41(95 \% \mathrm{CI}=0.16-1.00)$, while the adjusted hazard ratio for statins was 2.33 (95\% $\mathrm{CI}=1.40-3.87)$, in the low polypharmacy group.

When we conducted sensitivity analyses to assess bias due to deaths, we observed similar findings for the associations between each medication and function. However, among those with heart failure in the low polypharmacy group, the reduction in decline associated with RAS blockers lost significance when the risk of decline in the 27 deceased participants was assumed to range from $15 \%$ to $90 \%$. 


\section{DISCUSSION}

While there are multiple mechanisms by which the six guideline-recommended medication classes in this study may have either positive or negative effects on physical function, none of these medications was significantly associated with decline in physical function in the overall sample. However, among those in the low polypharmacy strata, RAS blockers were associated with a reduced risk of decline in those with heart failure, while statins were associated with an increased risk of decline in diabetics.

Some studies suggest that aldosterone blockade may reduce decline in physical function via muscle strength, but the majority of studies on the renin-angiotensin system have examined ACE inhibitor use (8). Two investigations provide some evidence of a reduction in disability, exercise capacity and muscle strength directly, and Simon et al., review studies showing a beneficial effect of ACEI/ARB on exercise capacity, although the exact mechanisms are unclear $(9,44,45)$. Our results indicated a protective association between RAS blockers and the risk of decline, but only in the low polypharmacy group. However, sensitivity analysis showed that if $\geq 15 \%$ of the decedents declined, then the protective association lost significance, suggesting this result is not robust to losses due to death.

For statins, studies examining various measures of physical function have mixed findings. Boheemen et al. (46) found statins were associated with better self-reported physical function, but not a summary performance measure of walking, chair rises, dressing and a tandem stand. Savo et al. review possible mechanisms suggesting that by reducing cardiovascular events, performance is maintained, in addition to the possibility that statins may reduce inflammation and the resultant impact on disability (10). However, Gray et al. found there was no association between statins and incident mobility limitation (walking one-quarter mile or climbing 10 steps without resting) over 6.5 years of follow-up (47). Our finding that there was an association of increased risk of decline in physical function for diabetic participants taking statins in the low polypharmacy group, while no difference in the high polypharmacy group deserves further study. Because we restricted our sample to participants with conditions (e.g. diabetes) indicated for prescribing statins while many studies use samples where participants may not have an indicated condition for statins, it is difficult to compare findings.

Finally, when we accounted for the deaths, the association between statins and increased risk of physical decline was still present, even when the outcome risk in decedents that was inflated from $14 \%-99 \%$.

There are strengths, as well as weaknesses, in using an observational design to examine the associations between medications and physical function. The large nationally representative sample of older adults with MCC provides information on a population often excluded from randomized controlled trials and the physical function outcomes are well-validated and of high importance to older adults, yet rarely considered in clinical trials. Medication use was ascertained by direct examination of containers.

We adjusted for a wide range of time-varying characteristics, including important medical conditions such as osteoarthritis, psychosocial measures and geriatric impairments which 
may influence prescribing the study medication and the functional outcomes. Multiple imputation analysis was used to address the potential bias due to missing data $(<1.9 \%)$ and we conducted a sensitivity analyses to assess the impact of losses due to death.

As in any observational study, we cannot infer causal relationships. Although there is adjustment for a wide range of covariates, we cannot eliminate the possibility of confounding by other unmeasured covariates that may bias our results. We also conducted several statistical tests, increasing the probability of finding an association by chance. We were limited to self-reported physical function, which reflects the participants' perceptions of their abilities and not necessarily an objective performance-based measure of function. We examined whether baseline polypharmacy exposure, measured using the number of concomitant medications, modified the associations between individual guidelinerecommended medications and physical function. However, number of medications alone cannot be used to inform prescribing decisions (48). The observed reduction in risk of decline in function associated with RAS blockers in the low polypharmacy group, could reflect a healthy user bias, as those taking fewer medications may be healthier and more likely to benefit from the medication. However, this would not explain the increase in risk associated with statins in those taking fewer medications. An additional limitation is that the significant interactions between polypharmacy, statins and RAS blockers were not specified a-prior so they should be interpreted with caution.

We could not include participants in Medicare Advantage plans as they do not have healthcare claims. Furthermore, our sample reflects higher-functioning older adults since we excluded those needing assistive devices were unable to perform the activities we assessed. This group is an important segment of the population of older adults and brings attention to the need to study universal cross-disease outcomes including perceived health, symptoms and social or recreational activities to determine benefits and harms of medications in this subgroup (49). Finally, we did not have information on when chronic conditions began and medication dose and treatment duration were unavailable.

Despite these limitations, our study suggests that with a few exceptions among those taking fewer concomitant medications, the use of guideline medications is not associated with decline in physical function in a higher functioning sample of older adults.

\section{Acknowledgments}

Funding:

This work was supported by grant R21 AG045148 and by the Yale Pepper Center (P30 AG021342), both from the National Institute on Aging. D. Gnjidic is supported by the Bridging Support Fellowship and International Profile Development Fund Grant. University of Sydney, Australia.

\section{References}

1. Gerteis, J., Izrael, D., Deitz, D., et al. Multiple Chronic Conditions Chartbook. Rockville, MD: Agency for Healthcare Research and Quality; 2014. [PDF - 10.62 MB] AHRQ Publications No, Q14-0038http://www.ahrq.gov/sites/default/files/wysiwyg/professionals/prevention-chronic-care/ decision/mcc/mccchartbook.pdf [Accessed September 12, 2016] 
2. Boyd CM, Darer J, Boult C, et al. Clinical practice guidelines and quality of care for older patients with multiple comorbid diseases: implications for pay for performance. JAMA. 2005; 294(6):716724. [PubMed: 16091574]

3. Ashman JJ, Beresovsky V. Multiple Chronic Conditions Among US Adults Who Visited Physician Offices: Data From the National Ambulatory Medical Care Survey, 2009. Prev Chronic Dis. 2013; 10:120308. [Accessed September 12, 2016] http://www.cdc.gov/pcd/issues/2013/12_0308a.htm.

4. Gnjidic D, Cumming RG, Le Couteur DG, et al. Drug Burden Index and physical function in older Australian men. Br J Clin Pharmacol. 2009; 68:97-105. [PubMed: 19660007]

5. Jyrkka J, Enlund H, Lavikainen P, et al. Association of polypharmacy with nutritional status, functional ability and cognitive capacity over a three-year period in an elderly population. Pharmacoepidemiology and drug safety. 2011; 20:514-522. [PubMed: 21308855]

6. Lorgunpai SJ, Grammas M, Lee DSH, et al. Potential Therapeutic Competition in CommunityLiving Older Adults in the U.S.: Use of Medications That May Adversely Affect a Coexisting Condition. PLoS ONE. 9(2):e89447.

7. Fried TR, Tinetti ME, Iannone L, et al. Health Outcome Prioritization as a Tool for Decision Making Among Older Persons with Multiple Chronic Conditions. Arch Intern Med. 2011; 171(20):18561858. [PubMed: 21965814]

8. Rich MW. Does aldosterone blockade improve physical performance in older individuals? Am J Med. 2013; 126(7):559-560. [PubMed: 23684063]

9. Simon CB, McMullen BL, Phelan D, et al. The renin-angiotensin system and prevention of agerelated functional decline: where are we now? Age. 2015; 37(13):1-11.

10. Savo A, Pierangela MM, Onder G, et al. Pharmacoepidemiology and disability in older adults: can medications slow the age-related decline in physical function? Expert Opin Pharmacother. 2003; 5(2):407-413.

11. Albert MA, Danielson E, Rifai N, et al. Effect of statin therapy on C - reactive protein levels; the pravastatin inflammation/CRP evaluation (PRINCE): A randomized trial and cohort study. JAMA. 2001; 286:64-70. [PubMed: 11434828]

12. Wu JH, Haan MN, Liang J, et al. Impact of antidiabetic medications on physical and cognitive functioning of older Mexican Americans with diabetes mellitus: A population-based cohort study. Ann Epidemiol. 2003; 13:369-376. [PubMed: 12821276]

13. Tomlinson SS, Mangione KK. Potential adverse effects of statins on muscle. Phy Ther. 2005; 85:459-465.

14. Forrest KYZ, Zmuda JM, Cauley JA. Patterns and determinants of muscle strength change with aging in older med. Aging Male. 2005; 8(3/4):151-156. [PubMed: 16390738]

15. Ortuno-Romeroa, R., O’Connell, M., Finucane, C., et al. [Accessed January 26, 2016] Insights into the clinical management of the syndrome of supine hypotension - orthostatic hypotension ( $\mathrm{SH}-$ $\mathrm{OH})$ : The Irish Longitudinal Study on Ageing (TILDA). Available at https:// bmcgeriatr.biomedcentral.com/articles/10.1186/1471-2318-13-73

16. Pacher P, Kecskemeti V. Cardiovascular side effects of new antidepressants and antipsychotics: new drugs old concerns? Curr Pharm Des. 2004; 10(20):2463-2475. [PubMed: 15320756]

17. Hilmer SN, Gnjidic D. The effects of polypharmacy in older adults. Clin Pharmacol Ther. 2009; 85(1):86-8. [PubMed: 19037203]

18. [Accessed June 8, 2015] Medicare Current Beneficiary Survey (MCBS). Centers for Medicare and Medicaid Services Website. http://www.cms.gov/Research-Statistics-Data-and-Systems/Research/ MCBS/index.html

19. Tinetti ME, McAvay G, Trentalange M, et al. Association between guideline-recommended drugs and death in older adults with multiple chronic conditions: population based cohort study. BMJ. 2015; 351:h4984. [PubMed: 26432468]

20. Allore HG, Zhan Y, Cohen AB, et al. Methodology to Estimate the Longitudinal Average Attributable Fraction of Guideline-recommended Medications for Death in Older Adults with Multiple Chronic Conditions. J Gerontol A Biol Sci Med Sci. 2016; 00:1-5.

21. Allore HG, Zhan Y, Tinetti ME, et al. Longitudinal average attributable fraction as a method for studying time-varying conditions and treatments on recurrent self-rated health: the case of 
medications in older adults with multiple chronic conditions. Ann Epidemiol. 2015; 25(9):681686. [PubMed: 26033374]

22. Piette JD, Kerr EA. The impact of comorbid chronic conditions on diabetes care. Diabetes Care. 2006; 29(3):725-731. [PubMed: 16505540]

23. Nagi SZ. An epidemiology of disability among adults in the United States. Milbank Mem Fund Q Health Soc. 1976; 54:439-67. [PubMed: 137366]

24. Nieves JW, Li T, Zion M, et al. The clinically meaningful change in physical performance scores in an elderly cohort. Aging Clin Exp Res. 2007; 19:484-491. [PubMed: 18172371]

25. Smith SC Jr, Benjamin EJ, Bonow RO, et al. AHA/ACCF secondary prevention and risk reduction therapy for patients with coronary and other atherosclerotic vascular disease: 2011 update: a guideline from the American Heart Association and American College of Cardiology Foundation. Circulation. 2011; 124(22):2458-73. [PubMed: 22052934]

26. Nathan DM, Buse JB, Davidson MB, et al. Medical management of hyperglycemia in Type 2 diabetes: a consensus algorithm for the initiation and adjustment of therapy: a consensus statement of the American Diabetes Association and the European Association for the Study of Diabetes. Diabetes Care. 2009; 32(1):193-203. [PubMed: 18945920]

27. January CT, Wann LS, Alpert JS. 2014 AHA/ACC/HRS guideline for the management of patients with atrial fibrillation: a report of the American College of Cardiology/American Heart Association Task Force on Practice Guidelines and the Heart Rhythm Society. Circulation. 2014; 130(23):2071-2104. [PubMed: 24682348]

28. Yancy CW, Jessup M, Bozkurt B, et al. 2013 ACCF/AHA guideline for the management of heart failure: a report of the American College of Cardiology Foundation/American Heart Association Task Force on Practice Guidelines. J Am Coll Cardiol. 2013; 62(16):e147-239. [PubMed: 23747642]

29. Work Group on Major Depressive Disorder. Practice guideline for the treatment of patients with major depressive disorder. 3. Arlington, V.A: American Psychiatric Publishing; 2010. http:// psychiatryonline.org/pb/assets/raw/sitewide/practice_guidelines/guidelines/mdd-guide.pdf [Accessed September 12, 2016]

30. Visser M, Goodpaster BH, Kritchevsky SB, et al. Muscle mass, muscle strength, and muscle fat infiltration as predictors of incident mobility limitations in well-functioning older persons. $\mathrm{J}$ Gerontol A Biol Sci. 2005; 60A:324-333.

31. Hernandez ME, Goldberg A, Alexander NB. Decreased muscle strength relates to self-reported stooping, crouching or kneeling difficulty in older adults. Phy Ther. 2010; 90(1):67-74.

32. Corsonello A, Garasto S, Abbatecola AM, et al. Targeting inflammation to slow or delay functional decline: where are we? Biogerontology. 2010; 11:603-614. [PubMed: 20549351]

33. Ferrucci L, Harris TB, Guralnik JM, et al. Serum IL-6 level and the development of disability in older persons. J Am Geriatr Soc. 1999; 47:639-646. [PubMed: 10366160]

34. Barzilay JI, Blaum C, Moore T, et al. Insulin resistance and inflammation as precursors of frailty: the Cardiovascular Health Study. Arch Intern Med. 2007; 167:635-641. [PubMed: 17420420]

35. Barzilay JI, Cotsonis GA, Walston J, et al. Insulin resistance is associated with decreased quadriceps muscle strength in nondiabetic adults aged $\geq 70$ years. Diabetes Care. 2009:736-738. VOLUME****. [PubMed: 19171728]

36. Donoghue OA, Jansen S, Dooley C, et al. Atrial fibrillation is associated with impaired mobility in community-dwelling older adults. SPELLING****JAMDA. 2014; 15(12):929-933.

37. Magnani JW, Wang MA, Benjamin EJ, et al. Atrial fibrillation and declining physical performance in older adults. The Health, Aging and Body Composition Study. Circ Arrhythm Electrophysiol. 2016; 9(5):e003525. [PubMed: 27052031]

38. Gangavati A, Hajjar I, Quach L, et al. Hypertension, orthostatic hypotension and the risk of falls in a community-dwelling elderly population: The maintenance of balance, independent living, intellect and zest in the Elderly of Boston Study. J Am Geriatr Soc. 2011; 59:383-389. [PubMed: 21391928]

39. Zicha SS, Tsuji Y, Takeshit-Shiroshita A, et al. Beta-blockers as antiarrthymic agents. Handb Exp Pharmacol. 2006; 171:235-266. 
40. Widen EI, Eriksson JG, Groop LC. Metformin normalizes nonoxidative glucose metabolism in insulin-resistant normoglycemic first-degree relatives of patients with NIDDM. Diabetes. 1992; 41:354-358. [PubMed: 1551495]

41. Stuck AE, Walthert JM, Nikolaus T, et al. Risk factors for functional status decline in communityliving elderly people: a systematic literature review. Soc Sci Med. 1999; 48:445-469. [PubMed: 10075171]

42. Quan H, Sundararajan V, Halfon P, et al. Coding algorithms for defining comorbidities in ICD-9CM and ICD-10 administrative data. Med Care. 2005; 43(11):1130-9. [PubMed: 16224307]

43. Yuan, Y. Sensitivity analysis in multiple imputation for missing data. Cary, NC: SAS Institute Inc; 2014. URL: https://support.sas.com/resources/papers/proceedings14/SAS270-2014.pdf [Accessed January 30, 2017]

44. Galindo-Ocana J, Bernabeu-Wittel M, Formiga F, et al. Effects of renin-angiotensin blockers/ inhibitors and statins on mortality and functional impairment in polypathological patients. Eur $\mathrm{J}$ Intern Med. 2012; 23:179-184. [PubMed: 22284251]

45. Coehlho VA, Probst VS, Nogari BM, et al. Angiotensin-II blockage, muscle strength, and exercise capacity in physically independent older adults. J Phys Ther Sci. 2016; 28:547-552. [PubMed: 27065543]

46. Boheemen LV, Tett SE, Sohl E, et al. Associations between statin use and physical function in older adults from the Netherlands and Australia: Longitudinal aging Amsterdam and Australian longitudinal study on women's health. Drugs Aging. 2016; 33(6):437-445. [PubMed: 27138957]

47. Gray SL, Boudreau RM, Newman AB, et al. Angiotensin-converting enzyme inhibitor and statin use and incident mobility limitation in community-dwelling older adults: The Health, Aging and Body Composition Study. J Am Geriatr Soc. 2011; 59:2226-2232. [PubMed: 22092102]

48. Gnjidic D, Tinetti M, Allore HG. Assessing medication burden and polypharmacy: finding the perfect measure. Expert Rev Clin Phar. 2017; 10(4):345-347.

49. Working Group on Health Outcomes for Older Persons with Multiple Chronic Conditions. J Am Geriatr Soc. 2012; 60:2333-2341. [PubMed: 23194184] 
Medication:

Condition:

Beta Blocker:

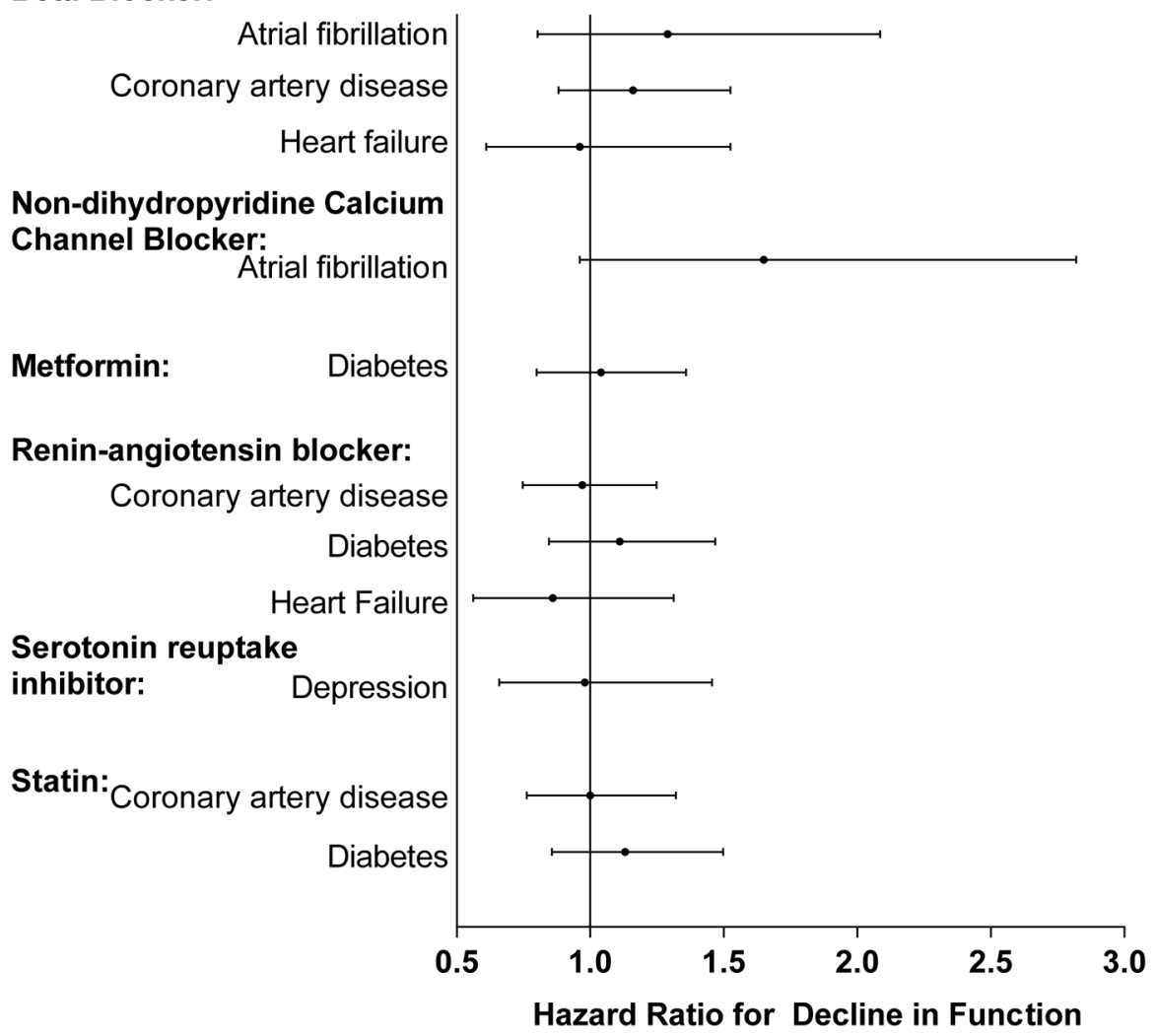

Figure 1. Adjusted Hazard Ratios for Decline in Physical Function by Guideline-Recommended Medication and Condition

The displayed hazard ratios reflect the effect of taking the medication for a specific condition versus not taking the medication and are adjusted for the covariates which included demographics, insurance, geriatric impairments, health behaviors, hospitalizations, medications (other guideline-recommended and number of concomitant), the other study conditions and Elixhauser comorbidity scale. Decline in physical function defined as a decrease in the number of activities (writing/handling objects, extending arms above shoulder, stooping/kneeling/crouching, lifting/carrying 10 pounds, walking $1 / 4$ mile or $2-3$ blocks) the participant was able to perform as compared to the baseline. Eight respondents who could not do any of the 5 activities were excluded since they could not decline. 


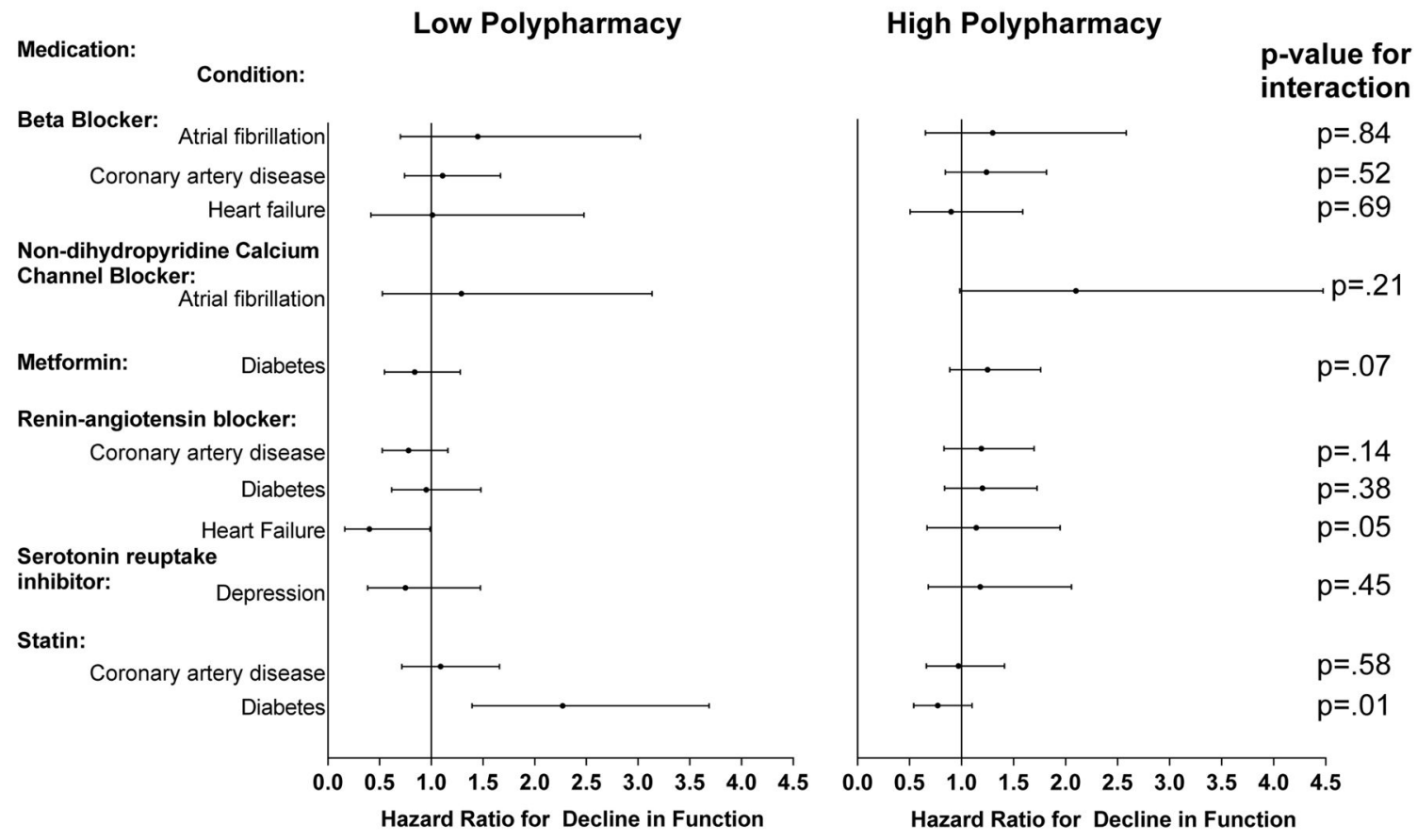

Figure 2. Adjusted Hazard Ratios for Decline in Physical Function by Guideline-Recommended Medication and Condition Stratified by Low and High Polypharmacy

The displayed hazard ratios reflect the effect of taking the medication for a specific condition versus not taking the medication and are adjusted for the covariates which included demographics, insurance, geriatric impairments, health behaviors, hospitalizations, medications (other guideline-recommended), the other study conditions and Elixhauser comorbidity scale. Decline in physical function defined as a decrease in the number of activities (writing/handling objects, extending arms above shoulder, stooping/kneeling/ crouching, lifting/carrying 10 pounds, walking $1 / 4$ mile or 2-3 blocks) the participant was able to perform as compared to the baseline. Eight respondents who could not do any of the 5 activities were excluded since they could not decline.

Low polypharmacy defined as $<7$ and High Polypharmacy as $>=7$ concomitant medications. 


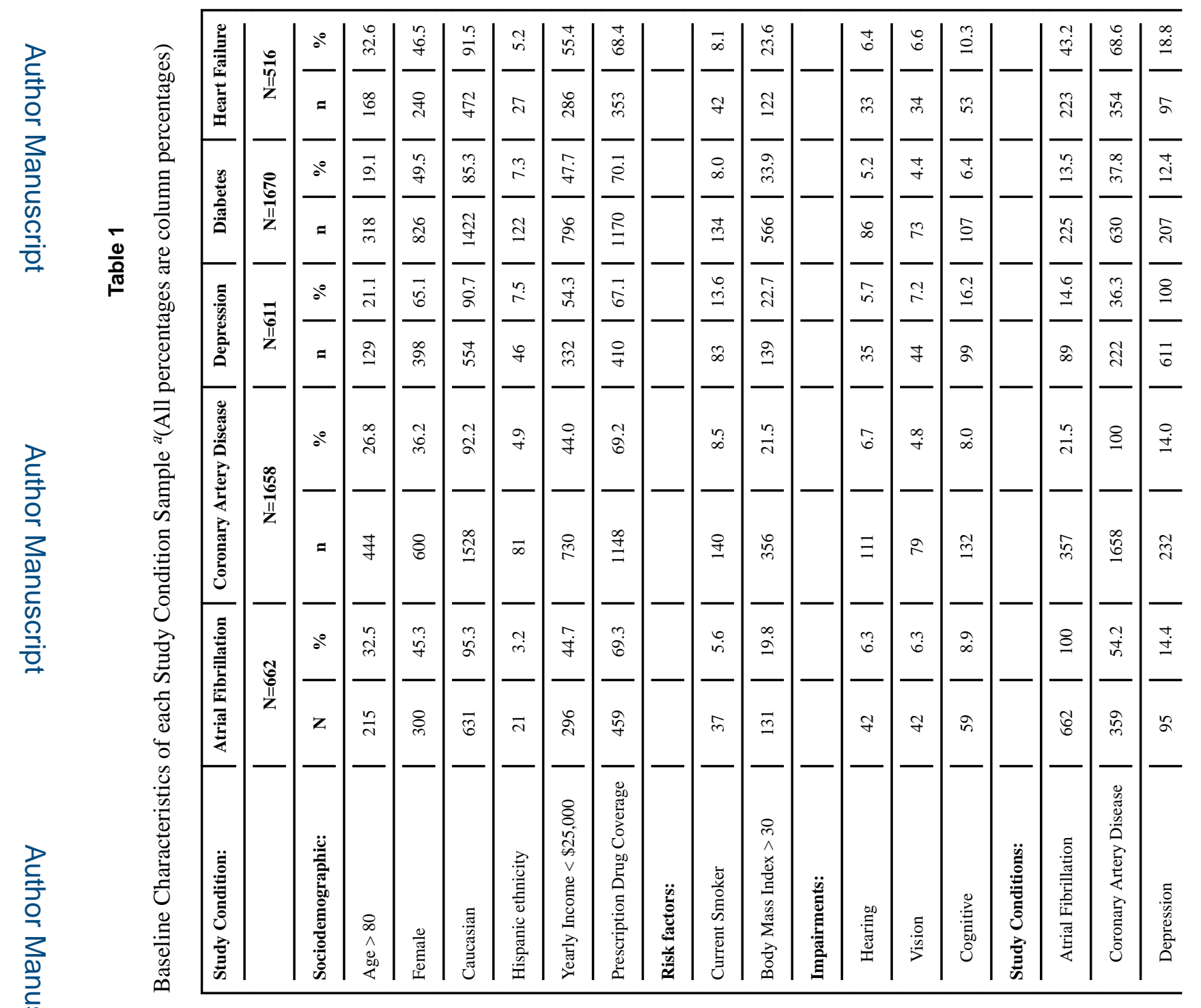




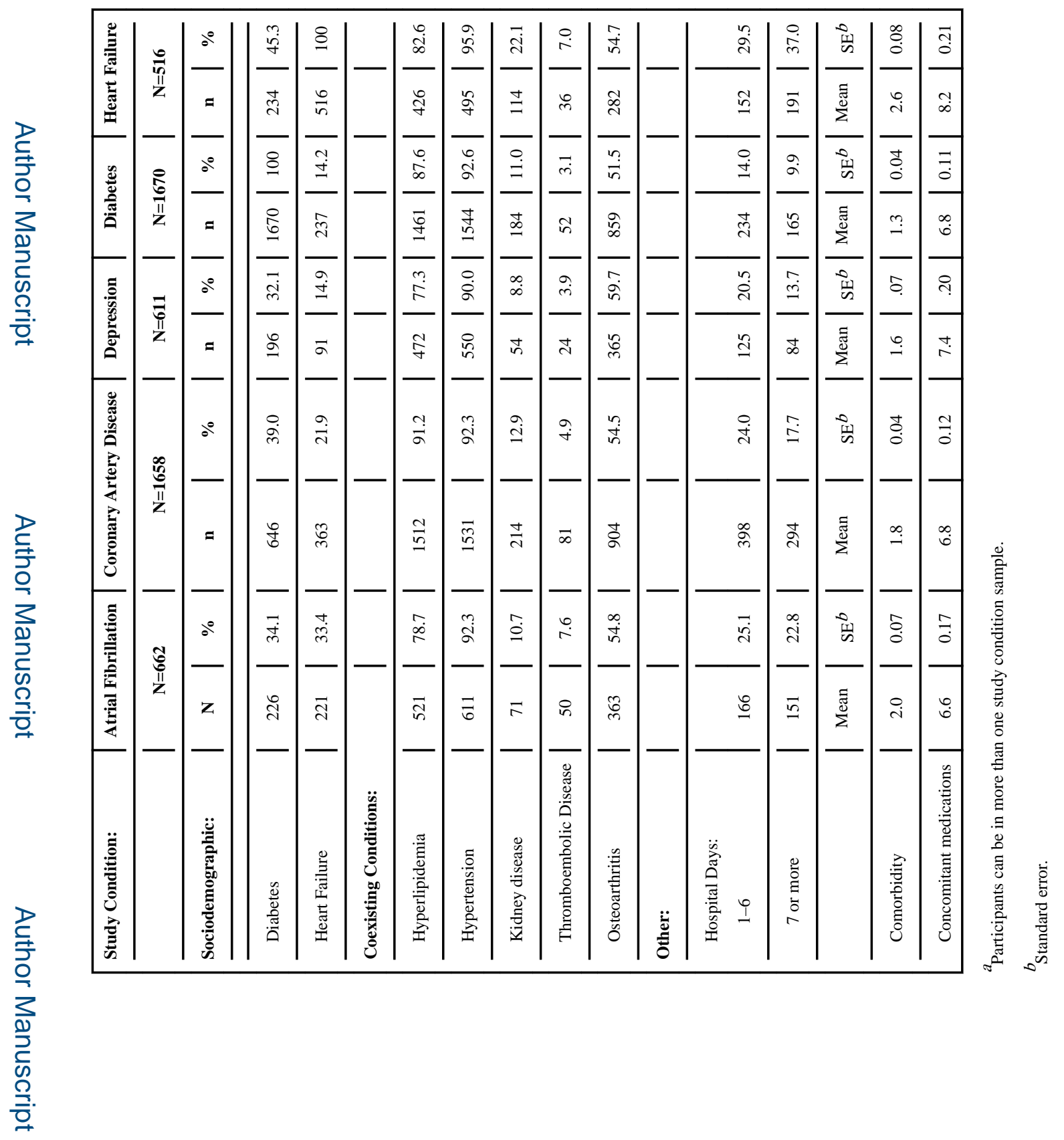

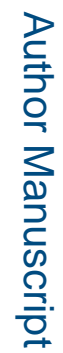



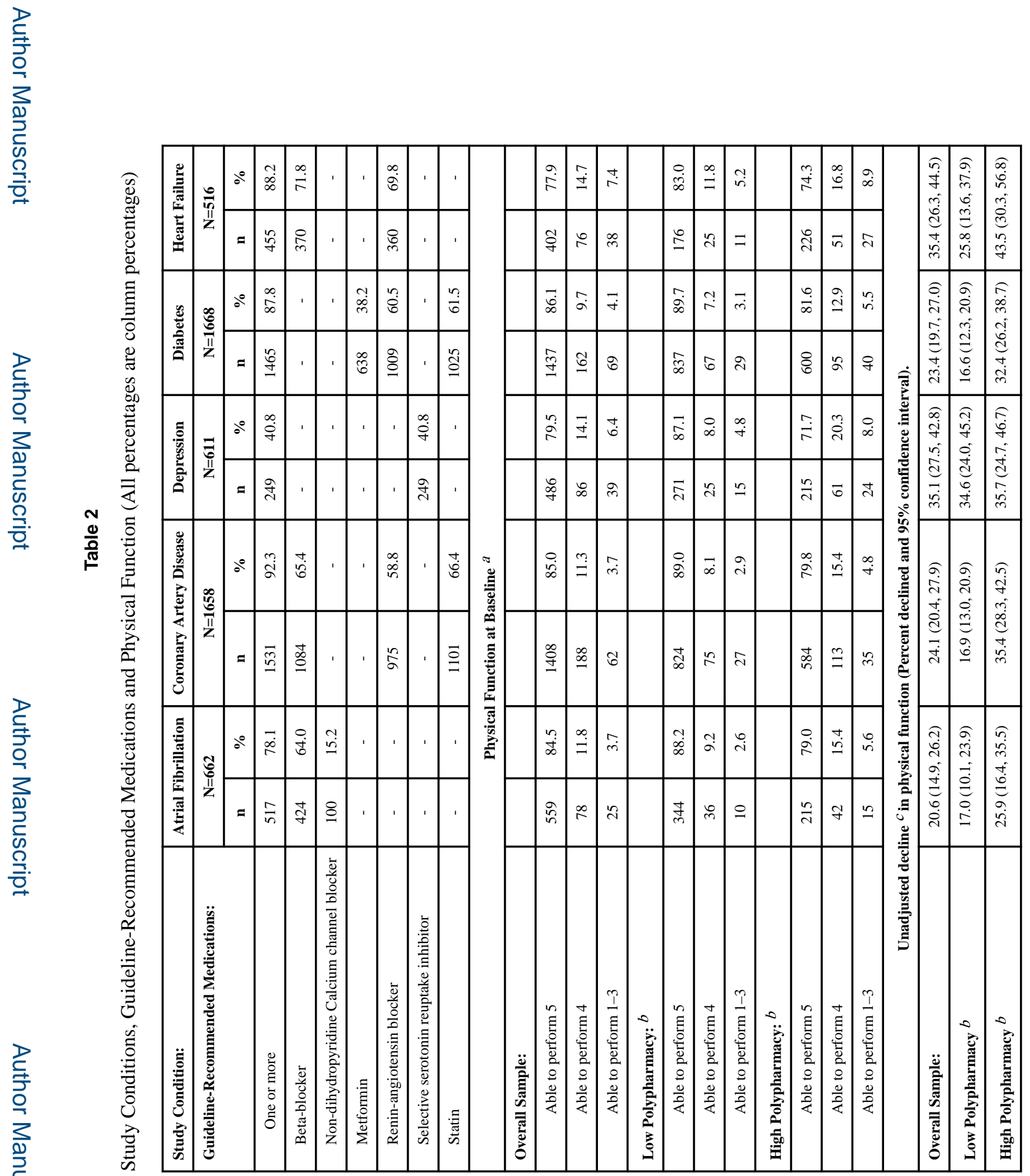

J Am Geriatr Soc. Author manuscript; available in PMC 2018 December 01 


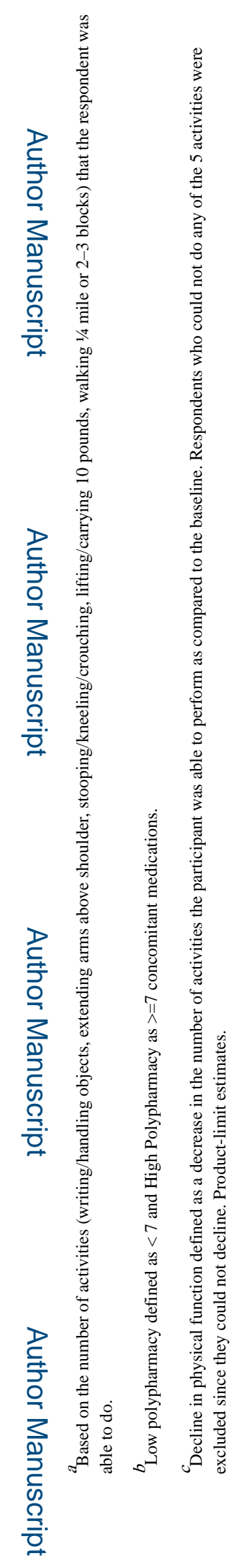

